\title{
Long-term exposure to leptin enhances the growth of prostate cancer cells
}

\author{
TERUTAKA NODA ${ }^{1}$, TADAHIKO KIKUGAWA ${ }^{1}$, NOZOMU TANJI ${ }^{1}$, NORIYOSHI MIURA ${ }^{1}$, \\ SEIJI ASAI ${ }^{1}$, SHIGEKI HIGASHIYAMA ${ }^{2,3}$ and MASAYOSHI YOKOYAMA ${ }^{1}$ \\ Departments of ${ }^{1}$ Urology, ${ }^{2}$ Biochemistry and Molecular Genetics and ${ }^{3}$ Cell Growth and Tumor Regulation, \\ Proteo-Medicine Research Center, Ehime University Graduate School of Medicine, Toon, Ehime 791-0295, Japan
}

Received November 6, 2014; Accepted December 23, 2014

DOI: $10.3892 /$ ijo.2015.2845

\begin{abstract}
Obesity correlates with an increased risk of developing prostate cancer (PCa) and leptin plays an important role in $\mathrm{PCa}$ progression. Since leptin is produced by adipocytes, the serum leptin level is higher in obese than in non-obese individuals. However, the effects of leptin remain controversial and unclear. The aim of the present study was to investigate the effect of leptin on PCa cell aggressiveness. Three human PCa cell lines (LNCaP, DU145 and PC-3) were treated with recombinant leptin for 28 days. Cell proliferation, migration, and invasion were estimated using the WST assay, a wound-healing assay, and a BD Matrigel invasion assay, respectively. The mechanism underlying the proliferative effect of leptin was investigated by cell transfections with small interfering RNA (siRNA) against the leptin receptor (ObR) or forkhead box O1 (FOXO1), and by immunocytochemistry. Long-term exposure of $\mathrm{PCa}$ cells to leptin enhanced their proliferation, migration and invasion. Leptin increased ObR expression and enhanced Akt phosphorylation constitutively. Leptin also increased the phosphorylation of FOXO1 via PI3K signaling and FOXO1 gene silencing enhanced PCa cell proliferation. Leptin induced the translocation of FOXO1 from the nucleus to the cytoplasm. Furthermore, the PI3K inhibitor, LY294002 suppressed this translocation. These results suggested that leptin regulated the subcellular localization of FOXO1 and induced Akt phosphorylation. Additionally, we revealed that leptin increased the expression of cyclin D1 and decreased the expression of $\mathrm{p} 21$ protein. In conclusion, long-term exposure to leptin increased the cell proliferation, migration, and invasion of PCa cells through inactivation of FOXO1. This inactivation resulted from exclusion of FOXO1 from the nucleus and its restriction to the cytoplasm through $\mathrm{PI} 3 \mathrm{~K} / \mathrm{Akt}$ signaling. Our
\end{abstract}

Correspondence to: Dr Terutaka Noda, Department of Urology, Ehime University Graduate School of Medicine, Shitsukawa, Toon, Ehime 791-0295, Japan

E-mail: notel3164@yahoo.co.jp

Key words: obesity, prostate cancer, leptin, FOXO1, signaling pathway findings contribute to an understanding of the association between obesity and PCa aggressiveness.

\section{Introduction}

In Japan, dietary changes favoring more Western eating habits have resulted in an increased rate of obesity (1). The prevalence of obesity in adult men has increased since the 1970 s, according to the data of the Japanese Ministry of Health, Labour and Welfare (Tokyo, Japan) (2). Obesity has become a major social and health issue. Obesity is related to an increased risk of not only metabolic diseases including cardiovascular disease, hypertension, and diabetes, but also of various cancers, particularly those with a hormonal basis such as breast and prostate cancer (PCa) (3). Indeed, the incidence of $\mathrm{PCa}$ has been increasing annually since the early 1990s in the Japanese population. PCa is predicted to become the second most common cancer among all cancers of Japanese men by 2020 (4). However, the relationship between PCa and obesity remains controversial, with some studies indicating that obesity is associated with a decreased incidence of PCa, and other studies suggesting an increased incidence and a worse survival among obese men (5-7). Several reports, including the comment by Freedland (8), have suggested that obesity might be more important as a risk factor for $\mathrm{PCa}$ progression rather than for its incidence.

Leptin, a $16-\mathrm{kDa}$ polypeptide adipokine that is secreted predominantly by adipose tissue, is considered to be involved in satiety regulation in humans and animals (9). In adults, circulating leptin levels are closely related to the amount of adipose tissue (10), so that serum leptin level is elevated in obese individuals compared to non-obese individuals. Leptin acts as a regulatory signal for the central nervous system, reflects adipose tissue stores, and influences energy homeostasis, eating behavior, appetite regulation and energy expenditure (11). Leptin also plays a role in reproduction, hematogenesis, neovascularization and cancer cell proliferation $(12,13)$.

Several studies have investigated the relationship between obesity, plasma leptin levels, and PCa. Chang et al (14) showed that men with elevated leptin levels had an increased risk of diagnosis with high-volume disease. It has also been shown that high leptin levels correlate with high stage and high grade 
of PCa (15). High expression levels of leptin and leptin receptor $(\mathrm{ObR})$, which is a member of the class I cytokine-receptor family, have been reported in prostate tissues (16); therefore, leptin may play an important role in the process of carcinogenesis and progression of prostate tumors.

Several recent in vitro studies have indicated that leptin acts as a mitogenic factor for various cell types including both normal and neoplastic cells $(17,18)$. ObRs have been identified in prostate cell membranes (19), and appear to function in activation of mitogenic signaling pathways $(20,21)$. In those studies, the proliferative effects of leptin on the androgen-sensitive (AS) PCa cell line, $\mathrm{LNCaP}$, were less pronounced than its effects on the androgen-resistant lines, DU145 and PC-3. In contrast to these reported proliferative effects of leptin on only androgen-insensitive (AI) cell lines, Deo et al have suggested that leptin induces the proliferation of only AS cell lines (22). They reported a dose-dependent increase in proliferation when LNCaP cells were cultured with leptin, but no changes in the growth of AI cells, even if they were incubated with a high dose of leptin. Thus, the effects of leptin on the growth of PCa cells remain controversial. In addition, the exact mechanism by which leptin exerts proliferative effects on cancer cells, including PCa cells, is not fully understood.

In the present study, we investigated the effects of leptin on the growth of both AS and AI PCa cells and on the underlying mechanisms of leptin action. Because exposure to elevated leptin levels for a long time is expected to result in higher $\mathrm{PCa}$ risk or higher PCa tumor aggressiveness in humans, the present study was performed using PCa cells that were cultured in medium containing various concentrations of leptin for 28 days.

\section{Materials and methods}

Materials. RPMI-1640 and Ham's F12 media for cell culture were supplied by Wako Pure Chemical Industries, Ltd. (Osaka, Japan) and Life Technologies (Carlsbad, CA, USA), respectively. Fetal bovine serum (FBS) was obtained from Nichirei Biosciences, Inc. (Tokyo, Japan). Monoclonal antibody against human $\beta$-actin and polyclonal rabbit antibodies against human ObR, and phosphorylated forkhead box O1 (FOXO1) (Ser256) were purchased from Sigma-Aldrich Japan K.K. (Tokyo, Japan) and Abcam (Tokyo, Japan), respectively. LY294002, used as an PI3K inhibitor, and polyclonal rabbit antibodies against phosphorylated Akt, Akt, cyclin D1, p21Cip1 and lamin A/C were obtained from Cell Signaling Technology, Inc. (Danvers, MA, USA). Polyclonal rabbit antibody against FOXO1 was obtained from Santa Cruz Biotechnology, Inc. (Santa Cruz, CA USA).

Cell culture and treatment. The AS human PCa cell line LNCaP and the AI human PCa cell lines DU145 and PC-3 used in this study were purchased from the American Type Culture Collection (ATCC) (Rockville, MD, USA). LNCaP and DU145 cells were maintained in RPMI-1640 medium and PC-3 cells were maintained in Ham's F-12 medium. Media were supplemented with $10 \%$ heat-inactivated FBS in the presence or absence of human recombinant leptin (Sigma Aldrich) at the indicated concentrations, in a humidified incubator at $37^{\circ} \mathrm{C}$ and $5 \% \mathrm{CO}_{2}$. The medium was changed every 4 days.
Cells were passaged when they were 50-70\% confluent. Cells were transferred into serum-free medium and cultured for $24 \mathrm{~h}$ under the same conditions except for the absence of serum.

Western blots. Cells were lysed with ice-cold lysis buffer and protease inhibitor and phosphatase inhibitor cocktails (Sigma-Aldrich). Samples were centrifuged at $12,000 \mathrm{x} \mathrm{g}$ for $10 \mathrm{~min}$ at $4^{\circ} \mathrm{C}$, and supernatants were electrophoresed on sodium dodecyl sulfate (SDS)-polyacrylamide gels and transferred to polyvinylidene difluoride membranes (Millipore, Bedford, MA, USA). After blocking with 5\% skimmed milk, the membranes were probed with primary antibodies overnight at $4^{\circ} \mathrm{C}$, followed by horseradish peroxidase-conjugated secondary antibody (GE Healthcare, Buckinghamshire, UK) for $1 \mathrm{~h}$ at room temperature. The immune complexes were visualized with the Enhanced Chemiluminescence Plus detection system (GE Healthcare) according to the manufacturer's instructions.

Gene silencing with siRNA. For small interfering RNA (siRNA) experiments, siRNA cocktails were obtained from, and functionally annotated by Cosmo Bio Co., Ltd. (Tokyo, Japan) and Bioneer, Inc. (Alameda, CA, USA). The siRNA cocktails are indicated in Table I. For the control, non-targeting siRNA (cat. no. 4390844) was obtained from Thermo Fisher Scientific, Inc. (Waltham, MA, USA). Cells were transiently transfected with each siRNA or control siRNA cocktail using the Neon Transfection System (Life Technologies). After 48-h incubation, cells were utilized for each assay.

Cell proliferation assay. The relative effect of leptin on the proliferation of each cell line was assessed using WST-8 assays and a Cell Counting kit-8 (Dojindo, Kumamoto, Japan). Cells that had been cultured in the presence or absence of graded concentrations of leptin for each day were re-seeded in 96-well plates at a density of $3 \times 10^{3}$ cells/well and were cultured in the same graded concentrations of leptin in at least three replicate wells at $37^{\circ} \mathrm{C}$ for $48 \mathrm{~h}$. The WST- 8 reagent was added to each well, and the cells were further incubated for another $3 \mathrm{~h}$. Absorbance was determined using a spectrophotometer (Thermo Scientific Multiskan FC; Thermo Fisher Scientific, Inc.) at $450 \mathrm{~nm}$. Absorbance values were expressed as percentages relative to untreated controls.

Cell invasion assay. The cell invasion assay was performed using a Matrigel Invasion Chamber from Becton-Dickinson (BD Biosciences, Tokyo, Japan) according to the manufacturer's instructions. Approximately $3 \times 10^{4}$ cells that had been cultured in the presence or absence of $100 \mathrm{ng} / \mathrm{ml}$ of leptin for 28 days were seeded onto the top of chamber and were incubated at $37^{\circ} \mathrm{C}$ in a $5 \% \mathrm{CO}_{2}$ incubator. After 48 -h incubation, the media were aspirated, and the cells remaining on the upper surface of the membrane and the Matrigel Matrix were removed with a sterile cotton swab. Invasive cells on the bottom surface of the membrane that had passed through the Matrigel were fixed in methanol and stained with Diff-Quik stain (Sysmex Corp., Hyōgo, Japan). The number of invaded cells on four representative sections of each membrane was counted in high-power fields under a light microscope (BX63; 
Table I. The siRNA used in this study.

\begin{tabular}{llc}
\hline siRNA & Sense $\left(5^{\prime} \rightarrow 3^{\prime}\right)$ & Antisense $\left(5^{\prime} \rightarrow 3^{\prime}\right)$ \\
\hline ObR & GGAAGAUGUAUGAGGUUUATT & UAAACCUCAUACAUCUUCCTT \\
& GGCUAGAUGGACUGGGAUATT & UAUCCCAGUCCAUCUAGCCTT \\
& CCAGAGUGAUGCAGGUUUATT & UAAACCUGCAUCACUCUGGTT \\
FOXO1 & GCUGCUGUAGAUAAGGACU & AGUCCUUAUCUACAGCAGC \\
& GUCCAAGACAUAGCUGGUU & AACCAGCUAUGUCUUGGAC \\
& CUGCAUAGCAUCAAGUCUU & AAGACUUGCUGCUAUGCAG
\end{tabular}

siRNA, small interfering RNA; ObR, leptin receptor; FOXO1, forkhead box O1.

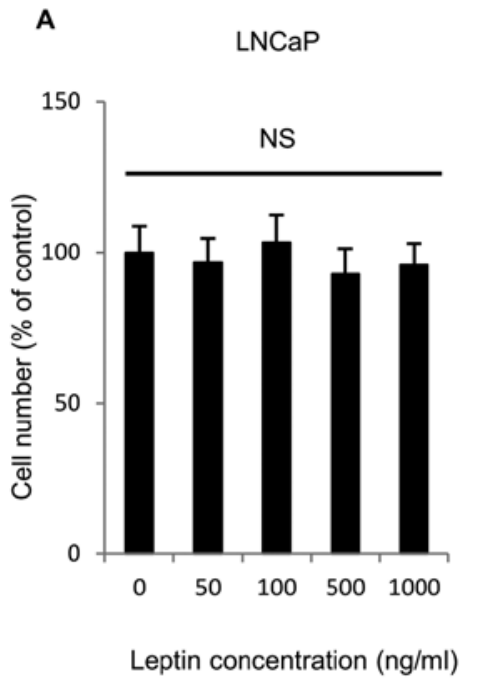

D

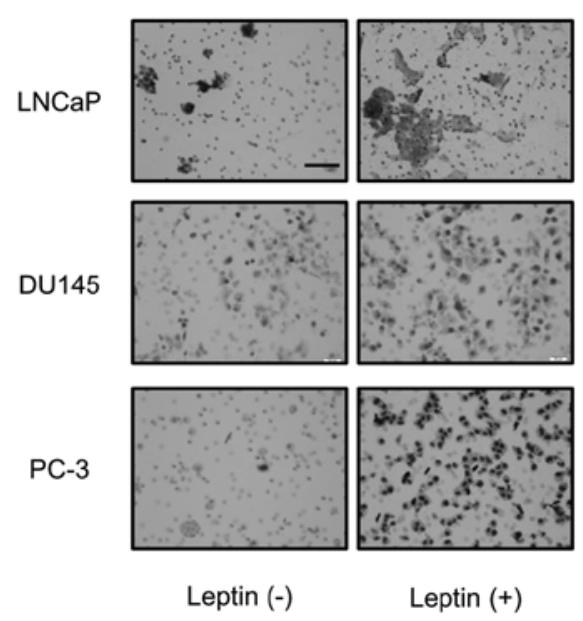

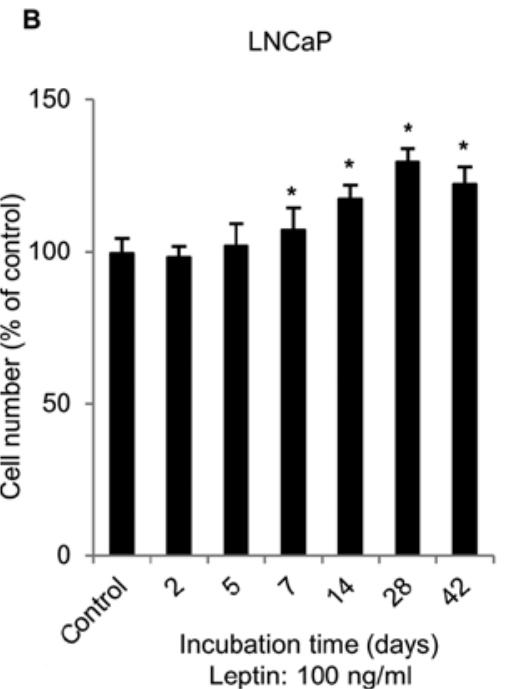

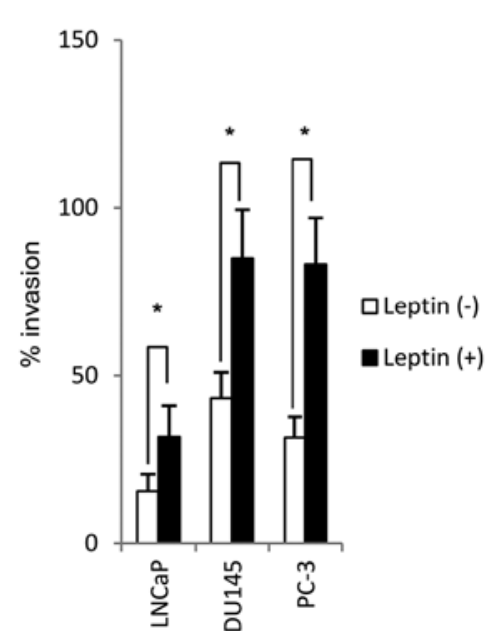

C

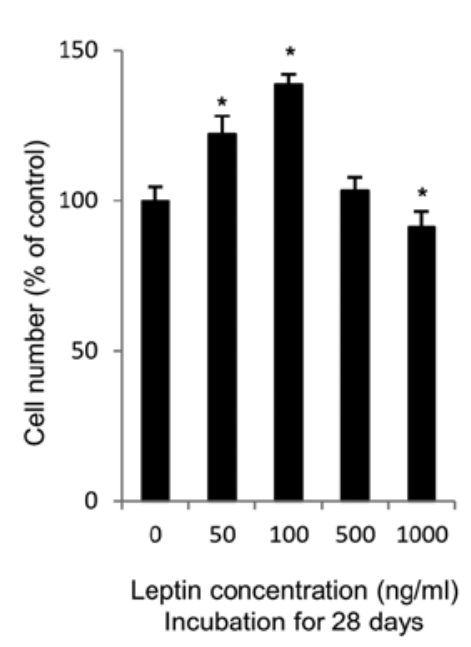

E

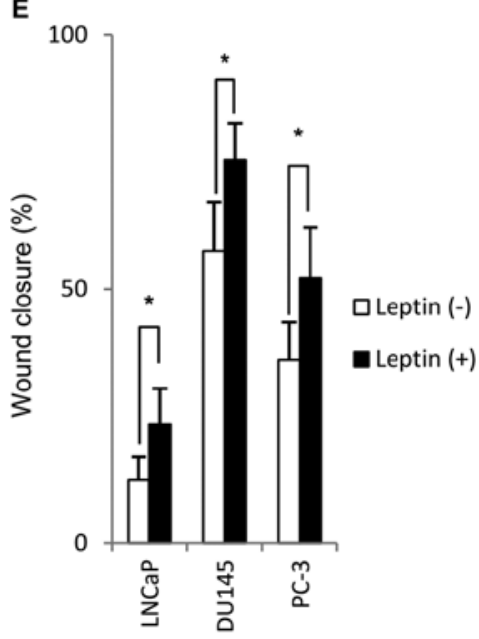

Figure 1. Effects of long-term leptin exposure on the proliferation, invasion, and migration of human prostate cancer (PCa) cells. (A) LNCaP cells were cultured in serum-free media for $48 \mathrm{~h}$ in the presence or absence of leptin $(0-1,000 \mathrm{ng} / \mathrm{ml})$ and cell numbers were determined using a colorimetric WST assay. (B) Time-course effects of proliferation of LNCaP cells cultured in the medium with $100 \mathrm{ng} / \mathrm{ml}$ leptin. (C) Effects of leptin at various concentrations for 28 days on the cell proliferation. (D) Cell invasion assay. The number of cells that invaded through the Matrigel was counted in four different regions. Photographs of representative images were taken at x 200 magnification. Scale bar, $100 \mu \mathrm{m}$. The data indicate percent invasion, i.e., the rates of the number of cells through Matrigel insert to control insert. (E) Scratch wound-healing assays. The reduction in the wound area was calculated using ImageJ. Values are expressed as means \pm standard deviation (SD). ${ }^{*} \mathrm{P}<0.05$.

Olympus Corp., Tokyo, Japan). Percent invasion, which was the percent of the seeded cells that had passed through the Matrigel, was calculated. The assay was repeated at least three times.
Scratch wound-healing migration assay. Cells that had been cultured in the presence or absence of $100 \mathrm{ng} / \mathrm{ml}$ of leptin for 28 days were seeded into 6-well plates and cultured in at least three replicate wells at $37^{\circ} \mathrm{C}$. When cells were grown to 
$90 \%$ or above confluence, a crossing linear scratch was made with a $200-\mu 1$ pipette tip, and floating cells were removed by washing with phosphate-buffered saline (PBS). These plates were photographed at the identical location both before and $18 \mathrm{~h}$ after incubation. The average area of the open scratch area was calculated using ImageJ ver $1.46 \mathrm{r}$ (NIH, Bethesda, MD, USA).

Immunofluorescence. Approximately $1 \times 10^{4}$ cells were seeded in a Lab-Tek II Chamber Slide (Thermo Fisher Scientific, Inc.). Cells were then further cultured in serum-free media for $48 \mathrm{~h}$ with the same leptin concentration. For PI3K inhibition, cells were pre-treated with LY294002 for $2 \mathrm{~h}$. The cells were then fixed with $4 \%$ paraformaldehyde and permeabilized with PBS containing $0.2 \%$ Triton $\mathrm{X}-100$ followed by blocking with $1 \%$ bovine serum albumin. Thereafter, the cells were incubated with polyclonal anti-FOXO1 antibody followed by incubation with fluorescein isothiocyanate-conjugated secondary antibody (Jackson ImmunoResearch Labs, West Grove, PA, USA). Trihydrochloride (Hoechst 33342; Dojindo) staining was used for nuclear detection. Fluorescence was photographed using an Olympus IX71 microscope (Olympus Corp.).

Subcellular fractionation. Subcellular fractionation was carried out using Nuclear/Cytosol Fractionation kits (BioVision, Inc., Milpitas, CA, USA) as described by the manufacturer. Equal volumes of each fraction were loaded onto an SDS-polyacrylamide gel, and western blotting was performed as previously described.

Statistical analysis. All values are expressed as the means \pm standard deviation (SD) of at least three independent experiments. Statistical analysis was performed using Student's unpaired t-test. $\mathrm{P}<0.05$ was considered statistically significant.

\section{Results}

Long-term exposure to leptin enhances PCa cell proliferation, invasion and migration. To clarify the effect of leptin on $\mathrm{PCa}$ cell proliferation, LNCaP, DU145 and PC-3 were treated with various concentrations of leptin for $48 \mathrm{~h}$. Fig. 1A-C shows only results derived from $\mathrm{LNCaP}$ cells, because the similar tendency was found in their experiments using three $\mathrm{PCa}$ cells examined. The incubation for $48 \mathrm{~h}$ did not affect the growth (Fig. 1A). Because short-term leptin exposure may not reflect the in vivo situation, we tried to examine the effects of long-term leptin exposure. Interestingly, time-course experiments showed that cell growth reached maximum after 28-day incubation with $100 \mathrm{ng} / \mathrm{ml}$ leptin (Fig. 1B). The most effective concentration of leptin was at $100 \mathrm{ng} / \mathrm{ml}$ (Fig. 1C), thus, $100 \mathrm{ng} / \mathrm{ml}$ was selected as the leptin concetration for further experiments. Maximum physiological concentration of serum leptin in human is $\sim 100 \mathrm{ng} / \mathrm{ml}$ (10). These results indicated that treatment with $100 \mathrm{ng} / \mathrm{ml}$ leptin for 28 days might be the relevant condition for induction of proliferation of all PCa cells. We tested the effect of long-term leptin treatment (1 month) on the ability of PCa cells to invade through Matrigel. As shown in Fig. 1D, PCa cells that were treated long-term with leptin showed significantly higher transit to the bottom surface of a Matrigel-coated membrane than non-leptin-treated cells.
In addition, the effect of long-term leptin treatment on the migration ability of PCa cells was estimated using a scratch wound-healing migration assay. As shown in Fig. $1 \mathrm{E}, \mathrm{PCa}$ cells treated long term with leptin showed a significantly larger healed area after scratching compared to non-treated cells. Thus, long-term exposure to leptin enhanced the malignant potential of PCa cell lines.

Long-term exposure to leptin increases ObR expression and Akt phosphorylation. Next, the effect of long-term leptin treatment on leptin-induced intracellular signaling pathways in PCa cells was investigated. We first determined if the ObR and intracellular Akt phosphorylation played a role in the enhanced proliferation observed in long-term leptin-treated cells. Akt phosphorylation was assayed because several groups have previously demonstrated that leptin enhances cell proliferation via the PI3K/Akt signaling pathway. Expression of the ObR, as assessed by western blotting, was significantly increased by long-term leptin treatment of all PCa cell lines, compared to non-treated cells, as shown in Fig. 2A. Furthermore, we confirmed that the level of Akt phosphorylation increased in all PCa cell lines that were treated with leptin for 1 month compared to non-treated cells.

ObR gene silencing and PI3K inhibitor suppress cell proliferation. To confirm that $\mathrm{ObR}$ signaling played a role in leptin-induced proliferation of PCa cells, ObR specific siRNA was transfected into PCa cells that had been exposed to leptin for 1 month. After 48-h transfection, ObR protein expression was markedly decreased in comparison to cells transfected with scrambled non-specific siRNA (Fig. 2B). The silencing of ObR significantly decreased the proliferation of all PCa cell lines compared to control siRNA cells. This result suggests that the ObR receptor mediates leptin effects on proliferation.

To further analyze the role of Akt activation in leptinenhanced PCa cell proliferation, we used LY294002 to inhibit Akt activation. Following long-term leptin culture, LY294002 or control dimethyl sulfoxide (DMSO) was added for $2 \mathrm{~h}$. And cells were incubated in serum-free media for $48 \mathrm{~h}$. As shown in Fig. 2C, inhibition of Akt phosphorylation with LY294002 decreased cell proliferation in the absence of leptin and further suppressed leptin-induced proliferation in LNCaP cells. We observed similar results in DU145 and PC-3 (data not shown). These results suggest that the PI3K/Akt signaling pathway is important for leptin-induced cell proliferation.

Long-term exposure to leptin induces phosphorylation of FOXO1 via the PI3K/Akt signaling pathway. The PI3K/Akt signaling pathway has been reported to phosphorylate FOXO1, which mediates some of its downstream signaling. To determine if FOXO1 was involved in leptin-mediated cellular effects, we first determined whether long-term exposure to leptin induces phosphorylation of FOXO1 using western blotting. As shown in Fig. 3A, long-term exposure to leptin induced a higher level of FOXO1 phosphorylation than that of non-treated cells. We further assayed if this phosphorylation is mediated by PI3K/Akt by assaying the effect of adding the specific PI3K inhibitor LY294002 $(25 \mu \mathrm{M})$ for the last $2 \mathrm{~h}$ of long-term leptin culture of PCa cells on FOXO1 phosphorylation. Following LY294002 treatment FOXO1 phosphorylation induced by leptin treatment 
A

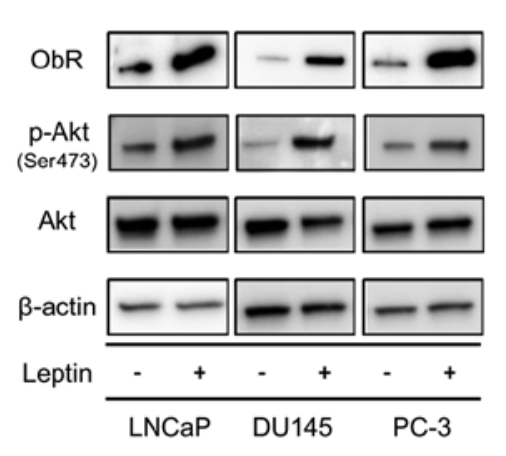

C

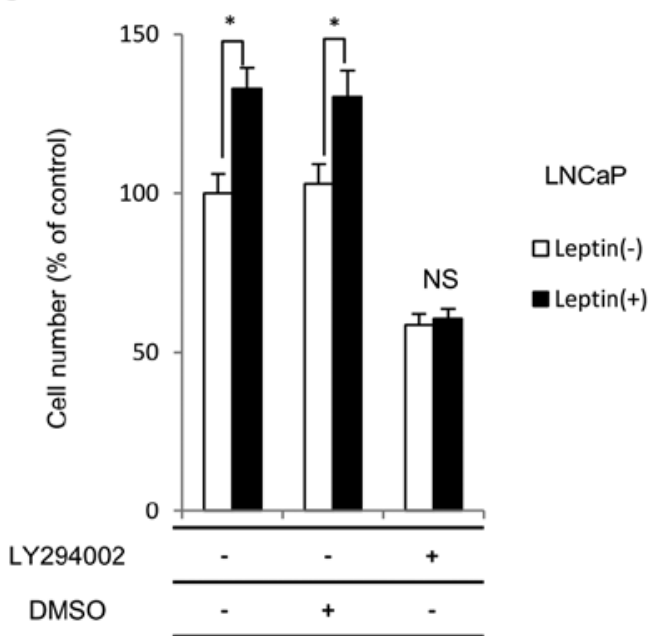

B
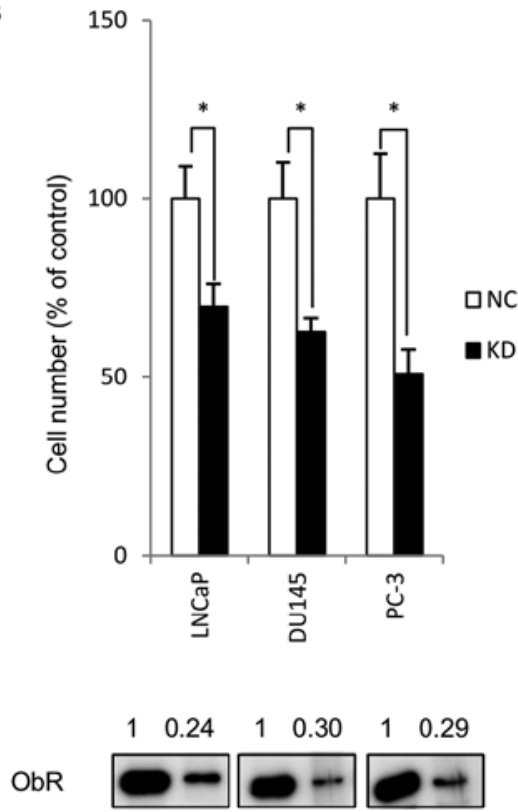

$\beta$-actin

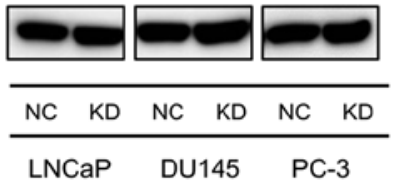

Figure 2. Participation of leptin receptor (ObR) and PI3K/Akt in human prostate cancer (PCa) cell proliferation. (A) Western blotting of the phosphorylation of Akt at Ser473 and of ObR expression. Akt protein and $\beta$-actin blots were used as loading controls. (B) Assay of the proliferation of leptin-treated PCa cells transfected with either ObR small interfering RNAs (siRNAs) (KD) or negative control siRNAs (NC) is shown. Numbers in the upper panel represent the average relative level of ObR expression, which is normalized for $\beta$-actin expression. (C) Assay of the proliferation of LNCaP cells treated with either a PI3K inhibitor or control dimethyl sulfoxide (DMSO) is shown. These cells were pre-treated with LY294002 (25 $\mu \mathrm{M})$ or DMSO for $2 \mathrm{~h}$. Values are expressed as means \pm standard deviation (SD). ${ }^{*} \mathrm{P}<0.05$.

A

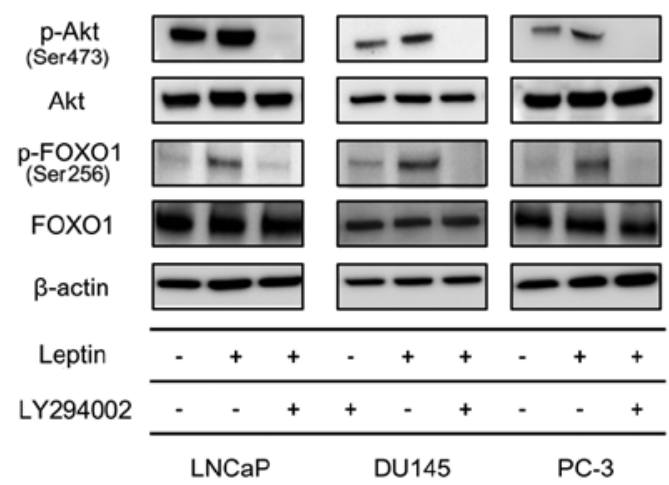

B
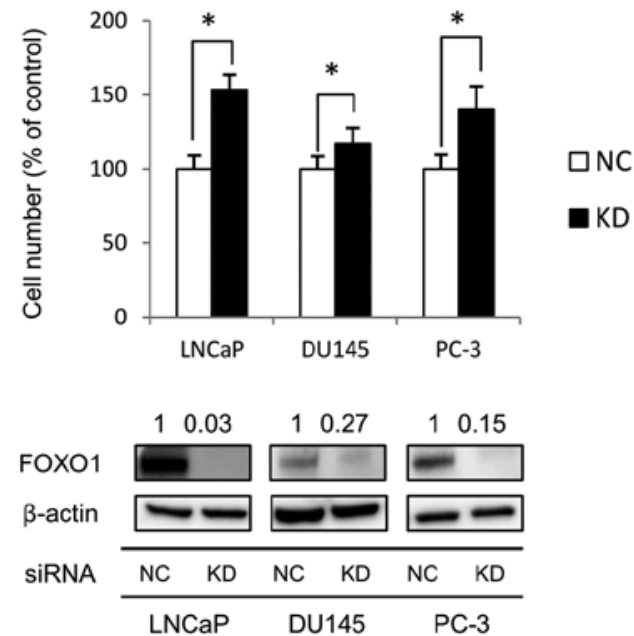

Figure 3. Role for forkhead box O1 (FOXO1) in human prostate cancer (PCa) cell proliferation. (A) Long-term leptin exposure induces FOXO1 phosphorylation that is inhibited by PI3K/Akt inhibition. For PI3K/Akt inhibition, $25 \mu \mathrm{M} \mathrm{LY} 294002$ was added for the last $2 \mathrm{~h}$ to cells with leptin. $\beta$-actin was used as a loading control. (B) FOXO1 downregulation enhances PCa cell proliferation. Leptin-untreated PCa cells were transfected with either FOXO1 small interfering RNAs (siRNAs) (KD) or negative control siRNAs (NC). Numbers in the upper panel represent the average relative level of FOXO1 expression which is normalized for $\beta$-actin expression. Values are expressed as means \pm standard deviation (SD). ${ }^{*} \mathrm{P}<0.05$. 


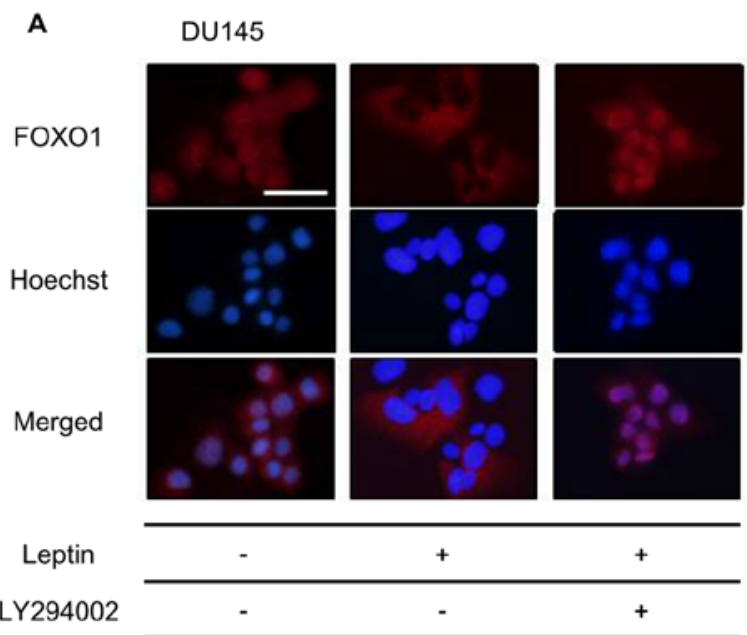

B
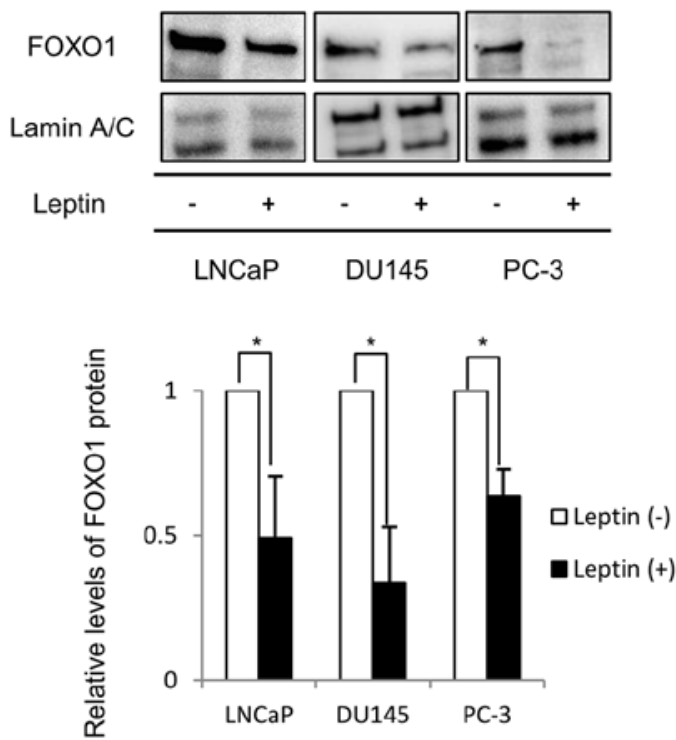

Figure 4. Effects of long-term exposure to leptin on intracellular localization of forkhead box O1 (FOXO1). (A) Immunocytochemical analysis of FOXO1. The PI3K inhibitor, LY294002 $(25 \mu \mathrm{M})$ was added into the medium containing leptin for the last $2 \mathrm{~h}$ of culture. FOXO1 is colored red. Cell nuclei are stained with the nucleic acid stain Hoechst (blue). Photographs of representative images were taken at x200 magnification. Scale bar, $50 \mu \mathrm{m}$. (B) FOXO1 in nuclear extracts of leptin-treated and -untreated cells. Lamin $\mathrm{A} / \mathrm{C}$ was used as a loading control of the nuclear fraction. Representative immunoblots and the quantification by scanning densitometry of three independent experiments are shown. Values are expressed as the means \pm standard deviation (SD). ${ }^{*} \mathrm{P}<0.05$.

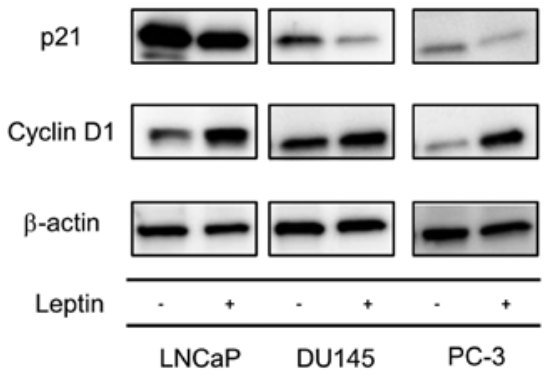

Figure 5. Effects of long-term leptin exposure on the expression of genes regulating the G1/S transition in human prostate cancer (PCa) cells. Lysates of human PCa cells treated with or without $100 \mathrm{ng} / \mathrm{ml}$ leptin for 1 month were immunoblotted using anti-cyclin D1 and p21Cip (p21) antibodies.

was greatly reduced compared to control cells (Fig. 3A). These results suggested that long-term exposure to leptin induced phosphorylation of FOXO1 via the PI3K/Akt signaling pathway.

FOXO1 gene silencing enhances $P C a$ cell proliferation. To clarify the role of FOXO1 in the proliferation of these $\mathrm{PCa}$ cell lines, we transiently transfected leptin-untreated cells with FOXO1 siRNA. After 48-h transfection, FOXO1 protein expression was decreased in comparison to cells transfected with scrambled non-specific siRNA (Fig. 3B). FOXO1 gene silencing significantly increased the proliferation of these cells compared to control cells (Fig. 3B). These results indicated that FOXO1 functions to suppress PCa cell proliferation.

Long-term exposure to leptin regulates nuclear/cytoplasmic localization of FOXO1 via PI3K. One possible mechanism by which Akt-mediated phosphorylation of FOXO1 might mediate FOXO1 effects on leptin-induced proliferation is by regulation of FOXO1 cellular localization. Thus, if FOXO1 is phosphorylated on specific residues by Akt, FOXO1 can be excluded from the nucleus, thereby inactivating its transcriptional activity. To confirm the effect of long-term exposure to leptin on the intracellular localization of FOXO1, immunocytochemistry was performed. As shown in Fig. 4A, FOXO1 was expressed in both the nucleus and the cytoplasm in DU145 cells. In contrast, FOXO1 expression in the nucleus was strongly decreased by long-term leptin treatment. The addition of LY294002 to the medium with leptin restored the intra-nuclear expression of FOXO1 protein. We observed similar results in LNCaP and PC-3 cells (data not shown). To further confirm the intracellular distribution of the FOXO1 protein, we compared FOXO1 expression in isolated nuclear fractions of leptin-treated and non-treated cells by western blotting. Fig. 4B shows that FOXO1 expression in the nuclei of leptin-treated cells was significantly decreased to $\sim 50 \%$ of that in non-treated cells.

Long-term exposure to leptin regulates the expression of cell cycle-related proteins. As described above, long-term exposure of leptin-induced PCa cell proliferation resulted from FOXO1 exclusion out of a nuclear region. We therefore next determined if the nuclear exclusion of FOXO1 that resulted from long-term exposure to leptin might affect the expression of cell cycle-related proteins. Increased cyclin D1 expression and decreased p21 expression was shown by western blotting of cells following long-term leptin treatment compared to non-treated cells (Fig. 5), suggesting that genes that regulate the G1/S transition were modulated towards cell cycle progression by leptin treatment.

\section{Discussion}

To our knowledge, this is the first report that long-term exposure of leptin increases proliferation, migration and invasion of PCa cells through inactivation of FOXO1. 
Most in vitro studies of leptin have only focused on analysis of the short-term effects of leptin exposure over 24-72 h. In preliminary studies we analyzed the effect of short-term leptin exposure on PCa cell proliferation and found that treatment with leptin concentrations of $0-1,000 \mathrm{ng} / \mathrm{ml}$ for $48 \mathrm{~h}$ did not modulate PCa cell proliferation (Fig. 1A). However, short-term leptin exposure may not reflect the in vivo situation. Thus, obese people are exposed to high levels of leptin over a long period. In addition, in humans, higher PCa risk or higher tumor aggression correlates with elevated leptin levels. For example, the study by Wang et al showed that a low-fat diet decreased the tumor growth of xenografted LNCaP cells (23). Another study demonstrated that a high-fat diet induced a high level of serum leptin and high argyrophilic nucleolar organizer region (AgNOR) counts in a transgenic adenocarcinoma of mouse prostate (TRAMP) model (24). These studies suggested that chronic hyperleptinemia might be related to $\mathrm{PCa}$ progression. The present study was therefore performed to clarify whether long-time exposure (28 days) to leptin affected the aggressiveness of three human PCa cell lines; LNCaP, DU145 and $\mathrm{PC}-3$, in vitro.

First of all, long-term exposure to leptin significantly enhanced the aggressiveness of all PCa cells. Leptin had a similar influence on both AS and AI PCa cells, although previous studies have not shown common effects of leptin on AS and AI PCa cells (20-22). In addition, we demonstrated that long-term exposure to leptin enhanced expression of the ObR and Akt phosphorylation. Overexpression of leptin and the expression of ObR have been reported to be associated with tumor progression in several types of cancers (25-28). ObR gene silencing resulted in a significant decrease in long-term leptin-induced cell proliferation. Moreover, the PI3K inhibitor also suppressed cell proliferation. These results suggested that the PI3K/Akt signaling pathway, via ObR, has an important role in leptin-induced proliferation.

Furthermore, we showed that the Akt phosphorylation induced FOXO1 phosphorylation. The PI3K/Akt signaling pathway is known to be important for FOXO1 phosphorylation (29). The FOXO family is composed of four members (FOXO1, 3a, 4, and 6) that are known to be important factors in many cellular processes including apoptosis, repair of damaged DNA, glucose metabolism and cell cycle arrest $(29,30)$. FOXO1 is known to function in the tumor suppression of several malignant tumors (31-35). Also in the present study, FOXO1 silencing promoted the proliferation of all PCa cell lines examined. Furthermore, FOXO1 has a role as a transcription factor in the nucleus that regulates a large spectrum of tumor suppression genes. The activities of FOXOs are controlled by post-translational modifications such as phosphorylation and acetylation $(29,30,36,37)$. Unphosphorylated FOXO proteins usually reside only in the nucleus. Once they are phosphorylated by intracellular environmental changes, they are exported to the cytoplasm and inactivated $(37,38)$. FOXO1 promotes cell cycle arrest in the nucleus via upregulation of cell cycle inhibitors or downregulation of cell cycle regulators $(29,30,39)$. We demonstrated that PCa cell proliferation induced by long-term exposure to leptin was a result of FOXO1 exclusion from the nucleus. In addition, increased cyclin D1 expression and decreased p21 expression was shown following long-term leptin treatment. These data suggest that leptin treatment may release these cells from cell cycle arrest, which FOXO1 regulates in the nucleus, by exclusion of FOXO1 from the nucleus. These findings closely match those of previous studies $(40,41)$, which indicate the induction of apoptosis through PI3K/FOXO1 signaling pathways. Lee et al have shown that MHY-449, a novel dihydrobenzofuro[4,5-b] naphthyridin-6-one derivative, induced apoptotic cell death in PC-3 cells through a reduction in the phosphorylation of Akt and FOXO1 and induced subcellular translocation of FOXO1 from the cytoplasm to the nucleus in these cells (41).

PCa growth depends mainly on androgen signaling via the androgen receptor (AR). Liu et al demonstrated that FOXO1 represses AR signaling, and that the nuclear localization of FOXO1 is necessary for its inhibition (42). Zhang et al also demonstrated that FOXO1 silencing attenuates methylselenic acid suppression of AR activity (33). Thus, in the case of AS PCa cells, leptin may increase AR activity by exclusion of FOXO1 from the nucleus.

In conclusion, our findings provide supporting evidence for a signaling pathway between long-term leptin treatment and FOXO1 function and contribute to an understanding of the association between obesity and PCa progression.

\section{Acknowledgements}

We are grateful to the members of the Higashiyama Laboratory (Ehime University Graduate School of Medicine, Ehime, Japan) for helpful suggestions. We thank Ms. Kazumi Kanno, Ms. Izumi Tanimoto, and Ms. Maria Mori for their excellent technical assistance.

\section{References}

1. Kaneko G, Miyajima A, Yuge K, Hasegawa M, Takeda T, Jinsaki M, Kikuchi E, Nakagawa K and Oya M: Periprostatic fat area is an independent factor that prolonged operative time in laparoscopic radical prostatectomy. Urology 82: 1304-1309, 2013.

2. Japanese Ministry of Health, Labour and Welfare: Results of Kokumin-Kenkou-Eiyou Chousa (in Japanese). http://www. mhlw.go.jp/.

3. Bray GA: The underlying basis for obesity: relationship to cancer. J Nutr 132 (Suppl 11): 3451S-3455S, 2002.

4. Utada M, Ohno Y, Soda M and Kamo K: Estimation of cancer incidence in Japan with an age-period-cohort mode. Asian Pac J Cancer Prev 11: 1235-1240, 2010.

5. Giovannucci E, Rimm EB, Liu Y, Leitzmann M, Wu K, Stampfer MJ and Willett WC: Body mass index and risk of prostate cancer in U.S. health professionals. J Natl Cancer Inst 95: 1240-1244, 2003.

6. Andersson SO, Wolk A, Bergström R, Adami HO, Engholm G, Englund A and Nyrén O: Body size and prostate cancer: a 20-year follow-up study among 135006 Swedish construction workers. J Natl Cancer Inst 89: 385-389, 1997.

7. Schuurman AG, Goldbohm RA, Dorant E and van den Brandt PA: Anthropometry in relation to prostate cancer risk in the Netherlands Cohort Study. Am J Epidemiol 151: 541-549, 2000.

8. Freedland SJ: Obesity and prostate cancer: a growing problem. Clin Cancer Res 11: 6763-6766, 2005.

9. Mistry AM, Swick AG and Romsos DR: Leptin rapidly lowers food intake and elevates metabolic rates in lean and ob/ob mice. J Nutr 127: 2065-2072, 1997.

10. Liuzzi A, Savia G, Tagliaferri M, Lucantoni R, Berselli ME, Petroni ML, De Medici C and Viberti GC: Serum leptin concentration in moderate and severe obesity: relationship with clinical, anthropometric and metabolic factors. Int J Obes Relat Metab Disord 23: 1066-1073, 1999.

11. Miller R, Tanofsky-Kraff M, Shomaker LB, et al: Serum leptin and loss of control eating in children and adolescents. Int $\mathrm{J}$ Obes (Lond) 38: 397-403, 2014. 
12. Sierra-Honigmann MR, Nath AK, Murakami C, GarcíaCardeña $\mathrm{G}$, et al: Biological action of leptin as an angiogenic factor. Science 281: 1683-1686, 1998.

13. Somasundar P, Yu AK, Vona-Davis L and McFadden DW: Differential effects of leptin on cancer in vitro. J Sur Res 113: 50-55, 2003.

14. Chang S, Hursting SD, Contois $\mathrm{JH}$, et al: Leptin and prostate cancer. Prostate 46: 62-67, 2001.

15. Tewari R, Rajender S, Natu SM, Goel A, Dalela D, Goel MM and Tondon P: Significance of obesity markers and adipocytokines in high grade and high stage prostate cancer in North Indian men - a cross-sectional study. Cytokine 63: 130-134, 2013.

16. Hoon Kim J, Lee SY, Myung SC, Kim YS, Kim TH and Kim MK: Clinical significance of the leptin and leptin receptor expressions in prostate tissues. Asian J Androl 10: 923-928, 2008.

17. Hardwick JC, Van Den Brink GR, Offerhaus GJ, Van Deventer SJ and Peppelenbosch MP: Leptin is a growth factor for colonic epithelial cells. Gastroenterology 121: 79-90, 2001.

18. Hoda MR, Keely SJ, Bertelsen LS, Junger WG, Dharmasena D and Barrett KE: Leptin acts as a mitogenic and antiapoptotic factor for colonic cancer cells. Br J Surg 94: 346-354, 2007.

19. Somasundar P, Frankenberry KA, Skinner H, et al: Prostate cancer cell proliferation is influenced by leptin. J Surg Res 118 $71-82,2004$.

20. Hoda MR and Popken G: Mitogenic and anti-apoptotic actions of adipocyte-derived hormone leptin in prostate cancer cells. BJU Int 102: 383-388, 2008

21. Onuma M, Bub JD, Rummel TL and Iwamoto Y: Prostate cancer cell-adipocyte interation: leptin mediates androgen-independent prostate cancer cell proliferation through c-Jun NH2-terminal kinase. J Biol Chem 278: 42660-42667, 2003.

22. Deo DD, Rao AP, Bose SS, et al: Differential effects of leptin on the invasive potential of androgen-dependent and -independent prostate carcinoma cells. J Biomed Biotechnol 2008: 163902, 2008.

23. Wang Y, Corr JG, Thaler HT, Tao Y, Fair WR and Heston WD: Decreased growth of established human prostate $\mathrm{LNCaP}$ tumors in nude mice fed a low-fat diet. J Natl Cancer Inst 87: 1456-1462, 1995.

24. Park SH, Chang SN, Baek MW, Kim DJ, Na YR, Seok SH, Lee BH, Kim KS and Park JH: Effects of dietary high fat on prostate intraepithelial neoplasia in TRAMP mice. Lab Anim Res 29: 39-47, 2013.

25. Garofalo C, Koda M, Cascio S, Sulkowska M, Kanczuga-Koda L, Golaszewska J, Russo A, Sulkowski S and Surmacz E: Increased expression of leptin and the leptin receptor as a marker of breast cancer progression: possible role of obesity-related stimuli. Clin Cancer Res 12: 1447-1453, 2006.

26. Ishikawa $M$, Kitayama $J$ and Nagawa $H$ : Expression pattern of leptin and leptin receptor (OB-R) in human gastric cancer. World J Gastroenterol 12: 5517-5522, 2006.

27. Riolfi M, Ferla R, Del Valle L, et al: Leptin and its receptor are overexpressed in brain tumors and correlate with the degree of malignancy. Brain Pathol 20: 481-489, 2010.

28. Howard JM, Beddy P, Ennis D, Keogan M, Pidgeon GP and Reynolds JV: Associations between leptin and adiponectin receptor upregulation, visceral obesity and tumour stage in oesophageal and junctional adenocarcinoma. Br J Surg 97: 1020-2027, 2010.
29. Zhang X, Tang N, Hadden TJ and Rishi AK: Akt, FoxO and regulation of apoptosis. Biochim Biophys Acta 1813: 1978-1986, 2011.

30. Huang $\mathrm{H}$ and Tindall DJ: Dynamic FoxO transcription factors. J Cell Sci 120: 2479-2487, 2007.

31. Li R, Erdamar S, Dai H, Wheeler TM, Frolov A, Scardino PT, Thompson TC and Ayala GE: Forkhead protein FKHR and its phosphorylated form p-FKHR in human prostate cancer. Hum Pathol 38: 1501-1507, 2007.

32. Zhang H, Pan Y, Zheng L, Choe C, Lindgren B, Jensen ED, Westendorf JJ, Cheng L and Huang H: FOXO1 inhibits Runx2 transcriptional activity and prostate cancer cell migration and invasion. Cancer Res 71: 3257-3267, 2011.

33. Zhang H, Fang J, Yao D, Wu Y, Ip C and Dong Y: Activation of FOXO1 is critical for the anticancer effect of methylseleninic acid in prostate cancer cells. Prostate 70: 1265-1273, 2010.

34. Scodelaro Bilbao P and Boland R: Extracellular ATP regulates FoxO family of transcription factors and cell cycle progression through PI3K/Akt in MCF-7 cells. Biochim Biophys Acta 1830: 4456-4469, 2013

35. Berry E, Hardt JL, Clardy J, Lurain JR and Kim JJ: Induction of apoptosis in endometrial cancer cells by psammaplysene A involves FOXO1. Gynecol Oncol 112: 331-336, 2009.

36. Rena G, Guo S, Cichy SC, Unterman TG and Cohen P: Phosphorylation of the transcription factor forkhead family member FKHR by protein kinase B. J Biol Chem 274: 17179-17183, 1999.

37. Kau TR, Schroeder F, Ramaswamy S, Wojciechowski CL, Zhao JJ, Roberts TM, Clardy J, Sellers WR and Silver PA: A chemical genetic screen identifies inhibitors of regulated nuclear export of a Forkhead transcription factor in PTEN-deficient tumor cells. Cancer Cell 4: 463-476, 2003.

38. Wen Q, Duan X, Liao R, Little P, Gao G, Jiang H, Lalit S, Quirion R and Zheng W: Characterization of intracellular translocation of Forkhead transcription factor O (FoxO) members induced by NGF in PC12 cells. Neurosci Lett 498: 31-36, 2011.

39. Wu Z, Sun H, Zeng W, He J and Mao X: Upregulation of MircoRNA-370 induces proliferation in human prostate cancer cells by downregulating the transcription factor FOXO1. PLoS One 7: e45825, 2012.

40. Chen Q, Ganapathy S, Singh KP, Shankar S and Srivastava RK: Resveratrol induces growth arrest and apoptosis through activation of FOXO transcription factors in prostate cancer cells. PLoS One 5: e15288, 2010.

41. Lee SH, Kang YJ, Sung B, et al: MHY-449, a novel dihydrobenzofuro[4,5-b][1,8] naphthyridin-6-one derivative, induces apoptotic cell death through modulation of $\mathrm{Akt} / \mathrm{FoxO1}$ and ERK signaling in PC3 human prostate cancer cells. Int J Oncol 44: 905-911, 2014.

42. Liu P, Li S, Gan L, Kao TP and Huang H: A transcription-independent function of FOXO1 in inhibition of androgen-independent activation of the androgen receptor in prostate cancer cells. Cancer Res 68: 10290-10299, 2008. 